\title{
Mobilidade urbana no programa ITgreen: perspectiva das Smart Cities
}

\section{Urban mobility in the ITgreen Plan: a perspective on Smart Cities}

WOJAHN, Rafaele Matte

Universidade Regional de Blumenau -FURB I rafaelewojahn@gmail.com

PRADA, Charles Anderson

Serviço Nacional de Aprendizagem Comercial SENAC-SC I charles.prada@gmail.com

MARTINEZ, Nadine Tamara

União Dinâmica de Faculdades Cataratas - UDC I nadi.mtz@gmail.com

FIALHO, Francisco Antonio Pereira

Universidade Federal de Santa Catarina - UFSC I fapfialho@gmail.com

\begin{abstract}
Resumo
Este estudo objetiva explanar as ações de mobilidade urbana no programa ITgreen no Porto Digital sob a perspectiva das Smart Cities. A pesquisa é de abordagem qualitativa, de estratégia de estudo de caso, de natureza analítica, e objeto de estudo o programa ITgreen. A pesquisa foi caracterizada como documental, utilizando-se de dados secundários. O eixo temático do Programa ITGreen trabalhado é o da Mobilidade Urbana, em consonância com um dos pilares das Smart Cities. Os resultados evidenciam que as ações praticadas visam tornar o perímetro urbano em que está instalado

\section{Abstract}

This study aims to explain the actions of urban mobility in the ITgreen Plan in Porto Digital, from the perspective of Smart Cities. The research uses a qualitative approach of analytical nature, and a case study in which the object is the ITgreen Plan. The research is also documentary, using secondary data sources. The thematic axis of the ITGreen Plan is Urban Mobility, in line with one of the pillars of support of Smart Cities. Results show that the actions performed in Porto Digital aim to transform that urban perimeter in a smart location/space/ neighborhood.
\end{abstract} o Porto Digital em um local/espaço/bairro inteligente.

Palavras-chave: Design thinking. Smart cities. ITgreen. Porto digital.

Keywords: Design thinking. Smart cities. ITgreen. Porto digital. 


\section{INTRODUC̣ÃO}

A transição do século XX para o século XXI foi marcada pela crescente urbanização (AL-NASRAWI; ADAMS; EL-ZAART, 2015). De acordo com o relatório World Urbanization Prospects 2014, emitido pelas Nações Unidas (UNITED NATIONS, 2014), é estimado para 2050 que 66\% da população ocupe em áreas urbanas. Este aumento tende a criar um ambiente complexo acerca das questões humanas e ambientais, incluindo o modo com que as pessoas vivem, se locomovem e utilizam serviços (ANTTIROIKO; VALKAMA; BAILEY, 2014). Com o aumento populacional dentro do perímetro urbano cabe as cidades proporcionarem boas condições de vida aos cidadãos atuais e futuros, envolvendo as dimensões econômica, tecnológica, social e regulatória (AL-NASRAWI; ADAMS; EL-ZAART, 2015).

Dentro desta perspectiva de melhorias urbanas, há como ferramenta a ser utilizada o design thinking voltado as melhorias urbanas, caracterizado como uma espécie de pensamento integrativo (BROWN, 2010). Como justificativa, muito antes de ser batizado como Design Thinking, as práticas utilizadas pelos designers para resolver problemas complexos, criar inovações e gerar qualidade de vida para as pessoas já eram utilizadas por diversos profissionais de várias áreas do conhecimento.

Um exemplo destes eventos é citado por Brown (2010), quando o autor menciona as obras desenvolvidas pelo engenheiro Isambard Kingdom Brunel, em referência principalmente a ferrovia Great Western Railway construída na Inglaterra, que pretendia proporcionar ao viajante "a melhor experiência possível". Estes e outros fatos fazem do design thinking tornar-se na última década a metodologia central a ser discutida por diversas áreas, justamente pelo seu aspecto multidisciplinar (SHAMIYEH, 2014). Assim, levando a construção do objetivo deste estudo: Explanar as ações de mobilidade urbana no programa ITgreen no Porto Digital sob a perspectiva das Smart Cities.

Para tanto, o artigo é constituído por cinco seções. A primeira caracteriza-se pela introdução, em que os temas centrais do artigo são apresentados, sendo, as smart cities e, o design thinking, bem como, o objetivo que se busca responder. A segunda seção corresponde a fundamentação teórica, compreendida pelo design thinking e pelas smart cities. Na terceira seção temse a descrição dos procedimentos metodológicos. Na quarta, a apresentação e análise dos resultados, iniciando por um tópico explicando o Porto Digital e, um sobre o programa ITgreen, implantado dentro do parque tecnológico Porto Digital. Na quinta seção têm-se as considerações finais, precedida pelas referências bibliográficas. 


\section{FUNDAMENTAC̣ÃO TEÓRICA}

Esta seção compreende a fundamentação teórica do artigo, subdividida em dois tópicos, sendo o primeiro correspondente ao design thinking, precedido pelas smart cities.

\subsection{Design Thinking}

O design thinking envolve um conjunto de métodos e técnicas que os designers utilizam conforme cita Brown (2010), mesmo que estes métodos e técnicas as vezes são originárias de outras áreas do conhecimento como: administração, economia, engenharia, antropologia, sociologia, entre outras.

Portanto, atualmente o Design Thinking está no centro do interesse do mundo acadêmico, empresarial e governamental, transformou-se conforme Shamiyeh (2014) disciplina obrigatória para todos explorarem o potencial das suas organizações e possibilitarem melhores resultados através da inovação e novas soluções que colocam as pessoas no centro, ou seja, as pessoas são as principais beneficiárias das soluções do Design Thinking.

Para Vianna et al. (2012) os benefícios do Design Thinking estão voltados para a visão holística empregada para as novas soluções, justamente por usufruir de vantagens proporcionadas pelo trabalho multidisciplinar das equipes, que entendem do que as pessoas precisam, cocriando com especialistas soluções e prototipando com o objetivo de melhorar o entendimento das necessidades afim de completar o conhecimento sobre o tema, com o resultado ao final propiciar soluções inovadoras.

Esta visão do todo que os designer thinkers - como são chamados os profissionais que utilizam habilidades dos designers - possuem, fazem com que as soluções propostas alcancem benefícios não só para a pessoas para qual a ideia foi sugerida, mas também para um grupo maior de pessoas, alcançado a comunidade.

Outro ponto importante, quando cita designer thinkers, não está se referindo somente designers por formação acadêmica e profissão, mas profissionais que conseguem utilizar, mesmo que empiricamente ou até as vezes inconsciente, habilidades que os designers são aprenderam ao longo do tempo (BROWN, 2010). Portanto, qualquer profissional poderá desenvolver e aplicar conhecimento, técnicas e métodos do Design Thinking, sendo esta uma das propostas das áreas de estudo, fazer com que todos os profissionais desenvolvam competências para que possam gerar soluções impactantes e direcionadas a qualidade de vida das pessoas. 
Brown (2010), ainda menciona que os profissionais devem ser do tipo $T$, ou seja, conseguem se comunicar de forma fluída, debatendo ideias, conceitos, pontos de vista com profissionais especialistas em áreas de conhecimento que não é sua área principal de formação.

Por fim, este é um dos grandes desafios atuais para o "novo", desenvolver soluções que geram mudanças e não somente para os patrocinadores através de retorno financeiro, mas sim alguma coisa diferente que cause impacto conforme Anthony (2012) quando este define inovação. O impacto que possa promover o desenvolvimento social, econômico, educacional e motivacional, para gerar uma engrenagem autossustentável sem a necessidade de fomento constante por organismos externos, como o governamental.

Quais são os mecanismos que o Design Thinking poderá agregar para obter soluções sofisticadas de problemas complexos para ambientes sociais? Esta questão pretende-se responder nos itens a seguir, citando os principais que os autores acreditam, sem esgotar as possibilidades do tema.

\subsubsection{Centrado no Ser Humano}

Quando Lockwood (2009) na sua definição do Design Thinking coloca no ser humano como o principal beneficiário de um processo de inovação isto enfatiza a importância das pessoas para as abordagens que envolvem o Design Thinking. Esta importância segundo Brown (2010) é realizada através da:

a) Observação: a necessidade sempre de sair a campo para poder coletar as informações com maior qualidade possível sobre as pessoas que estão envolvidas direta ou indiretamente na solução desejável. Estas informações são úteis para entender e verificar as novas possibilidades que serão disponibilizadas para poder propor a melhor solução para determinado problema.

b) Insights: $O$ insight não vem de dados quantitativos, o insights vem das observações, das experiências, do conhecimento, com isso, a partida para ter insigths é sair pelo mundo e observar as verdadeiras experiências das pessoas.

c) Empatia: é se colocar no lugar das pessoas, do ser humano, é fazer a conexão com as pessoas que são o foco das observações em nível profundo. Segundo Brown (2010) é o hábito mental que nos leva a pensar nas pessoas como pessoas.

Além do fator humano, está na forma de criar novas soluções, na busca de alternativas para posteriormente convergir na melhor proposta, através do pensamento divergente e convergente. 


\subsubsection{Pensamento divergente e pensamento convergente}

Pensamento divergente e convergente procura fazer com que a equipe quando está a procura por soluções deixe a zona de conforto e desenvolve o óbvio, o incremental conforme cita Brown (2010). Para um melhor entendimento pode-se explanar um exemplo: imagina na sua cidade uma grande área que precisa ser ocupa ou revitalizada, qual é a alternativa óbvia? Conceder a área através de permuta, vender ou qualquer outro acordo para a iniciativa privada construir um conjunto de edifícios residenciais, comerciais, ou outra opção qualquer. Este é o óbvio, é o incremental.

Uma equipe trabalhando na forma de pensamento divergente e convergente, irá primeiro criar um conjunto de opções, ou seja, divergir. Portanto, o processo inicial está baseado na maneira de criar opções, novas ideias e geração de alternativas, quanto mais melhor, mesmo que mais possibilidades de escolha resulte em uma complexidade maior do processo conforme Brown (2010) enfatiza, reconhecendo que a tarefa de criar opções não seja fácil, todos por natureza são suscetíveis e tendenciosos a trabalhar com o óbvio, é mais fácil.

O pensamento convergente é fazer escolhas, tomar decisões, verificar na quantidade de possibilidades qual é a melhor alternativa para um problema ou necessidade. Para Brown (2010, p. 62) "o pensamento convergente é uma forma prática de decidir entre alternativas existentes".

No entanto é necessário analisar as restrições impostas pelo novo que são trabalhadas pelo Design Thinking: desejável, factível e viável. Pois não adiantar adotar uma alternativa que não atenda estas características.

\subsubsection{Desejável, factível e viável}

Existe uma importância fundamental em toda a essência abordada pelo Design Thinking, a existência de fatores que podem gerar uma solução não uniforme, sem equilíbrio, aquela solução que atenda uma necessidade, mas desencadeia problemas colaterais muito maiores que os benefícios promovidos pelo novo.

Com isso, segundo Brown (2010, p. 18 grifo nosso) "a disposição e até a aceitação das restrições constituem o fundamento do Design Thinking", que nos estágios iniciais são identificados critérios que se sobrepõe uns aos outros. Estes critérios estão agrupados em restrições denominadas: desejável, factível e viável, conforme mostra a Figura 1. 
Figura 1 - O equilíbrio das restrições, fator essencial para a melhor solução.

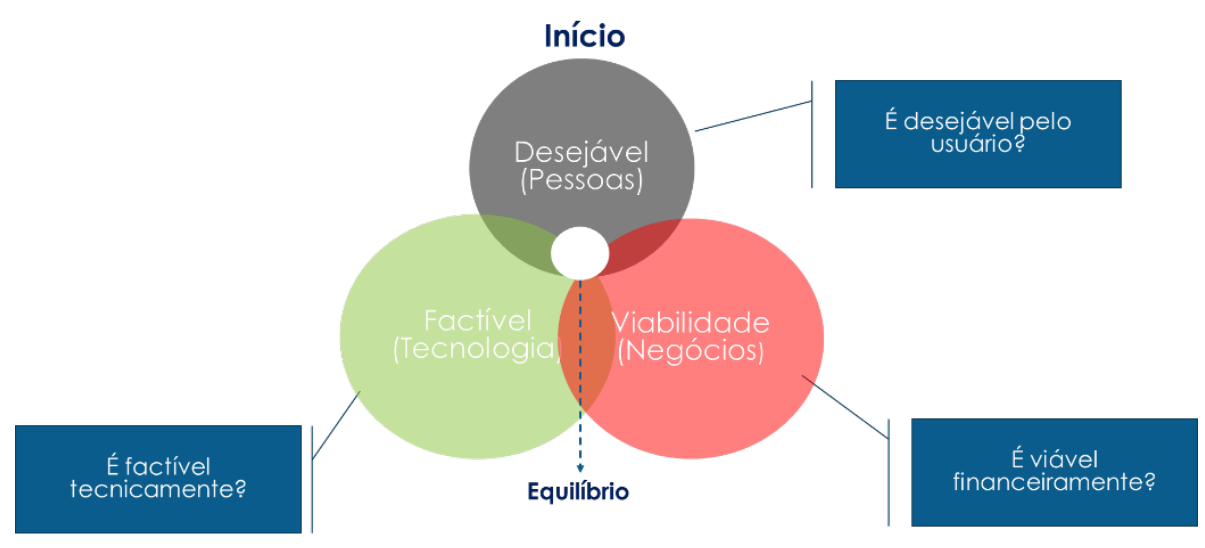

Fonte: Adaptado de Brown (2010).

As restrições significam (i) desejabilidade (analisar se faz sentido para as pessoas); (ii) praticabilidade (verificar se é funcionalmente possível num futuro próximo) e, (iii) viabilidade (como tornará parte de um modelo de negócios sustentável).

Como mostra a Figura 1, a necessidade da busca pelo equilíbrio harmonioso entre estas três restrições. Portanto um profissional que utiliza as habilidades de designer buscará a solução de sucesso que contemple todas essas três restrições. Para testar a solução, primeiro desenvolverá uma série de protótipos utilizando a prototipagem, conforme item a seguir.

\subsubsection{Prototipagem}

A prototipação pode ser muito além do que tangibilizar uma ideia ou conceito, poderá validar, testar e levantar novas informações para verificar se o caminho que se tomará com a solução é o mais correto.

Segundo Vianna et al. (2012) a necessidade de desenvolver um protótipo se faz coerente para tangibilizar uma ideia, para o autor é a passagem do abstrato para o físico de forma a representar a realidade em esfera simplificada, mas importante para realizar as validações.

Ainda segundo Vianna et al. (2012) é um instrumento de aprendizado sob dois aspectos, o da ótica da equipe do projeto e, o do ponto de vista do usuário. O primeiro instrumento para realizar a fidelidade da solução e o segundo para poder interagir e assim fornecer insumos para a evolução do protótipo.

Para David Kelley, fundador da IDEO, prototipagem é "pensar com as mãos", protótipos devem ser rápidos e sujos como sugere Brown (2010), 
para não consumirem muito esforço e recurso, testar e evoluir o quanto antes o conceito, para que ao final a solução de um problema complexo tenha uma simplicidade para facilitar a implementar decorrente do protótipo.

\subsection{Smart Cities}

Com a intensa urbanização há a perda de funcionalidades básicas afetando significativamente a qualidade de vida da população, mas ao mesmo tempo representam desafios para a gestão pública (WEISS; BERNARDES; CONSONI, 2015). Tais perdas podem ser enfrentadas com o aproveitamento adequado das capacidades atuais e futuras, melhorando a eficiência e reinventando a organização das cidades, tendo as TICs como viabilizadoras de um sistema nervoso para e de cidades inteligentes.

O conceito de cidade inteligente é amplamente conectado com as tecnologias da informação e da comunicação (TICs). Para Anttiroiko, Valkama e Bailey (2014) uma cidade inteligente renova a ideia de comunidade local em que há uma congruência entre governo, empresas e residentes que usam TICs para três fins principais, sendo os serviços econômicos, geração de emprego e qualidade de vida, além de reforçar o papel da comunidade.

Giffinger et al. (2007) elencou seis pilares das cidades inteligentes, sendo economia, pessoas, governança, mobilidade, meio ambiente e vida inteligente, construídas sob uma combinação inteligente de cidadãos engajados e independentes.

Embora o cerne das cidades inteligentes sejam as soluções tecnológicas (ANTTIROIKO; VALKAMA; BAILEY, 2014), o desempenho urbano não depende apenas da dotação de capital físico, mas também da disponibilidade e qualidade da comunicação do conhecimento do capital humano e social (CARAGLIU; DEL BO; NIJKAMP, 2011). A dimensão humana de uma cidade inteligente é tratada como pessoas inteligentes (NAM; PARDO, 2011). Giffinger et al. (2007) ainda abordam o cosmopolitanismo, flexibilidade, criatividade, mente aberta, participação na vida pública, entre outros.

A governança inteligente foca a participação baseada em arranjos organizacionais e em instituições democráticas, em uma parceria integrativa entre a comunidade e as autoridades que busca ir além da adoção das TICS (ANTTIROIKO; VALKAMA; BAILEY, 2014).

Para a economia inteligente, o The Committee of Digital and KnowledgeBased Cities (UCLG, 2012) adota o conceito de cidades que possuem indústrias inteligentes, como as que produzem TICs ou que envolvem as TICs em seus processos de produção. Giffinger e Gudrun (2010) reconhecem como atributos 
de uma cidade com economia inteligente as que possuem espírito inovador, empreendedores, produtividade, habilidade de transformação, entre outros.

A mobilidade inteligente compreende oferecer aos cidadãos novas tecnologias no sentido de mobilidade que permitam facilitar a vida urbana (UCLG, 2012). Giffinger e Gudrun (2010) propõe a integração entre as TICs e os modais de transporte. O lugar ou vida inteligente é relacionado a fatores como facilidades culturais, saúde, segurança, facilidades no quesito da educação e coesão social (GIFFINGER et al., 2007). O The Committee of Digital and Knowledge-Based Cities (UCLG, 2012) adota dois eixos, um sendo o e-health e, acessibilidade e e-inclusão.

Diante do exposto, as cidades inteligente representam um fenômeno global que convergem para o desenvolvimento socioeconômico e, não manifestando-se apenas em casos isolados (NAM; PARDO, 2011), entretanto, nesses casos são respeitadas as características de um sistema urbano sustentável, de modo a contribuir com a qualidade de vida e bem-estar social dos cidadãos que ali realizam as suas necessidades.

\section{MÉTODOS E TÉCNICAS DE PESQUISA}

Buscado responder ao objetivo geral deste estudo, optou-se conduzir a pesquisa pela abordagem qualitativa, utilizando a estratégia de estudo de caso, de natureza analítica e, tendo como objeto de estudo o programa ITgreen, desenvolvido pelo Porto Digital. A abordagem qualitativa objetiva exploração, bem como, entendimento e significado dado a determinados problemas sociais (CRESWELL, 2010).

Relacionado ao estudo de caso, este se refere à exploração de um evento em profundidade (CRESWELL, 2010), constituindo-se de um estudo aprofundado de um dado fenômeno social (BABBIE, 2014). Quanto a natureza analítica, trata da análise do fenômeno que constitui o estudo de caso, por meio da construção da explanação sobre o fenômeno (YIN, 2010). Assim, este estudo foi conduzido sob tal abordagem por que visa o entendimento das ações do projeto ITgreen sob a perspectiva das Smart Cities no tocante a soluções de mobilidade urbana, discutidas em profundidade.

Para a coleta de dados, a pesquisa foi caracterizada como pesquisa documental, cujo dados foram coletados em fontes secundárias. Para tanto, foram consultados documentos institucionais fornecidos pelo Porto Digital e, dados coletados em dois sítios, o sítio institucional do Porto Digital e, o sítio do projeto ITgreen. Quanto a análise dos dados, foram observados e adaptados 
os passos propostos por Creswell (2010), sendo organização e preparação dos dados, leitura dos dados, codificação dos dados por tema de análise, interpretação dos dados e, interpretação da análise.

Embora o Programa ITgreen opere em quatro eixos temáticos, sendo a) resíduos tecnológicos; b) formação de jovens; c) acessibilidade digital e, d) mobilidade urbana, para este estudo de caso foi escolhido trabalhar com um específico, sendo o de mobilidade urbana. Tal escolha deu-se por esse eixo apresentar melhorias aparentes na qualidade de vida dos cidadãos que permeiam o Porto Digital e estar em consonância com um dos pilares que envolve as Smart Cities. Ademais, este programa, já vem apresentando resultados concretos e desdobramentos no tocante a melhorias, conforme será apresentado na próxima seção, condizente a análise dos dados.

\section{ANÁLISE DOS DADOS}

Esta seção compreende a análise dos dados do estudo. Para tanto, o primeiro item corresponde a compreensão do Porto Digital e, o segundo item compreende ao programa ITgreen, focalizando nas ações realizadas pelo programa, bem como, as evidências dos pilares correspondentes as Smart Cities.

\subsection{O Porto Digital}

O Porto Digital é um parque tecnológico, localizado na cidade de Recife, estado de Pernambuco, instalado em uma região histórica e urbana. Sua atuação está voltado para duas principais atividades: software e serviços de tecnologias da informação e comunicação, como também atividades relativas a economia criativa (COSTA, 2014).

A trajetória dentre a tecnologia da informação e da comunicação faz do Porto Digital um local em que há a predisposição para economia inteligente. Em conformidade com o The Committee of Digital and Knowledge-Based Cities (UCLG, 2012), cidades que possuem economia inteligente investem em indústrias inteligentes, como as que envolvem ou produzem TICs.

A história do Porto Digital inicia quando um grupo de professores da Universidade Federal de Pernambuco (UFPE) notou a necessidade de criar condições para a permanência dos profissionais formados no estado, pois existia na época uma grande quantidade de mão-de-obra qualificada migrando para outras regiões do Brasil. Com isso, no final dos anos 90 surgiu o Centro de 
Estudos e Sistemas Avançados do Recife (CESAR), com o objetivo de fomentar e criar novos empreendimentos de tecnologia no estado (AMORIM; AMORIM, 2004).

O início do Porto Digital foi modesto, ocupando espaço reduzido e com apenas duas empresas / instituições constituindo o parque tecnológico. Atualmente é um centro pujante de ideias, inovação, empreendedorismo e formação de profissionais qualificados, com aproximadamente 259 empresas / instituições abrigadas em $1.810 .000 \mathrm{~m}^{2}, 800$ empreendedores e 8.500 profissionais trabalhando diretamente em organizações situadas no Porto Digital (PORTO DIGITAL, 2015a).

Portanto, o Porto Digital é um dos casos de sucesso para uma nova transformação do novo, deixando para trás um pensamento linear, onde o óbvio não foi a opção primária e sim a melhor solução, mesmo sendo desafiante, como foi.

\subsection{O Programa ITgreen}

Conceitualmente, o Porto Digital define o ITgreen como um "[...] programa do Porto Digital voltado para a promoção das tecnologias da informação para o desenvolvimento socioambiental" (PORTO DIGITAL, Março 2015). Para tanto, o programa possui quatro eixos específicos em que suas ações são desenvolvidas, sendo "[...] (i) Resíduos Tecnológicos; (ii) Acessibilidade Digital; (iii) Formação para inclusão de jovens no mercado de trabalho; e (iv) Cidades Sustentáveis" (PORTO DIGITAL, Março 2015).

O Porto Digital tem a consciência de que o desenvolvimento tecnológico e econômico não pode estar dissociado do desenvolvimento social e da preservação ambiental. Por essa razão, desenvolve junto as suas empresas, ações estruturadas de responsabilidade social" (PORTO DIGITAL, Março 2015). O conceito de cidade inteligente sustentável trata-se de uma cidade inovadora que usa os recursos da TICs visando a melhoria na qualidade de vida, operações e serviços urbanos eficientes, competitividade, no intuito de garantir aspectos econômicos, sociais e ambientais na presente e futuras gerações ( $A L$ NASRAWI; ADAMS; EL-ZAART, 2015).

A ausência de planejamento urbano em algumas cidades faz com que elas cresçam de forma desordenada, principalmente quando se trata de países em desenvolvimento, é neste papel que as TICs, quando usadas de forma inteligente estão inseridas, com o propósito de tornar os locais mais seguros (UCLG, 2012), com investimento em capital humano que visa melhorar a 
qualidade de vida (NEIROTTI et al., 2014). Estas contribuições trazidas pelo programa vão encontro das palavras de Brown (2010), quando este menciona que o aspecto social deve ser ressaltado.

A Mobilidade Urbana no Porto Digital atua por meio de outro projeto, o PortoLeve, que busca agregar o uso de TICs com vistas à sustentabilidade aos serviços urbanos, visando melhorias para mobilidade e segurança, bem como, na imagem do parque tecnológico Porto Digital, buscando tornar "[...] o ambiente como um lugar de referência na implantação e testes de tecnologias inovadoras; melhorar a qualidade de vida das pessoas; além de contribuir para tornar o Porto Digital uma região inteligente e fazer Recife uma cidade inteligente" (ITGREEN, [2016?]).

Os resultados obtidos pelo programa são o (i) sistema de compartilhamento de bicicletas; (ii) realização do moBIT (Seminário Internacional de Mobilidade); e, (iii) criação da revista moBIT (ITGREEN, [2016?]).

Observa-se ações buscando oferecer serviços de qualidade para as pessoas (NAM; PARDO, 2011), além das soluções de mobilidade urbana. Para tanto, o programa busca o desenvolvimento das TICs, debate, fomento e busca de informações voltadas a mobilidade urbana e ao desenvolvimento sustentável (ITGREEN, [2016?]). Para Okuda el al. (2012), a mobilidade urbana deve ser constituída de um sistema equilibrado que objetive a sustentabilidade, também nesse meio.

O sistema de compartilhamento de bicicletas, chamado de Porto Bike, que estimula o uso do meio de transporte para distâncias pequenas, oriundo de uma parceria entre o poder público (Ministério da Ciência, Tecnologia e Inovação e o Governo do Estado de Pernambuco) e o Porto Digital (PORTOLEVE, [2016?]). O programa foi implementado em 2013, distribuiu 100 bicicletas em 10 estações, perfazendo a interligação do Bairro do Recife, São José e Santo Amaro (ITGREEN, [2016?]; PORTOLEVE, [2016?]). Esta parte do programa possibilita

[...] não apenas uma nova alternativa de deslocamento, mas a proposta de um olhar diferente para a cidade, ao ar livre e sobre duas rodas. Além de estimular a mudança de comportamento das pessoas, melhorar a convivência entre o carro e a bicicleta, desafogar o trânsito, ainda reduz a emissão de poluentes. $O$ projeto também conta com tecnologias sustentáveis, uma vez que as estações são movidas a energia solar (ITGREEN, [2016?]). 
Quanto aos objetivos do projeto é a introdução da bicicleta como um modal de transporte sustentável, buscando construir hábitos saudáveis, redução da emissão de poluentes, humanizar o perímetro urbano e promover a responsabilidade social nas pessoas (PORTOLEVE, [2016?]). O sítio do PortoLeve apresenta outras funcionalidades como o carro elétrico, denominado Carro Leve, zona azul eletrônica, vagas de estacionamento disponível por meio de aplicativo, informações sobre os modais de transporte público e, posicionado com a informação "em breve", estacionamentos inteligentes (PORTOLEVE, [2016?]).

Semelhante ao Porto Bike, o Carro Leve, é um serviço de aluguel de carros elétricos (PORTOLEVE, [2016?]). São três veículos disponíveis e cinco estações dentro do bairro do Recife em um sistema visa economia, mobilidade e sustentabilidade, ativado por aplicativo e integrado aos demais modais de transporte (PORTOLEVE, [2016?]).

O pilar mobilidade inteligente trata de situações semelhantes a estas, tanto da criação de um sistema de modais de transporte que seja considerado eficiente e confortável, quanto da criação de uma estrutura que atenda a necessidade dos cidadãos, no intuito da otimização deste tipo de serviço (OKUDA et al., 2012).

Outros serviços oferecidos pelo PortoLeve ([2016?]) são a zona azul eletrônica permite que o motorista ative por meio de um aplicativo, cujo download pode ser realizado no sítio do Porto Leve, um cartão virtual de estacionamento, disponível para o perímetro do Porto Digital e, consulta de vagas de estacionamento (PORTOLEVE, [2016?]).

No quesito segurança, também há a projeção da implantação de um sistema de gestão de veículos, em parceria com o DETRAN/PE, cujo intuito é identificar a circulação de veículos no bairro, identificação das principais rotas de circulação e, estatísticas (PORTOLEVE, [2016?]). Quanto aos modais de transporte disponíveis no parque tecnológico, o Porto Leve criou uma plataforma em que é possível realizar a consulta destes, bem como, os serviços por eles oferecidos (PORTOLEVE, [2016?]).

Para Giffinger e Gudrun (2010) o pilar "mobilidade inteligente" deve fazer a ligação entre os sistemas de informação e de comunicação e, os de transporte, sendo que um dos fatores a serem considerados é a disponibilidade de uma estrutura para este fim. Essa integração é encontrada dentro do Porto Digital e, é uma das preocupações, bem como, desenvolvimento do programa ITGreen.

Outra solução de mobilidade urbana, pode ser visualizada a seguir, ainda que descrita como "em breve" não deixa de ser uma solução com potencial para ser implantada. O projeto do Porto Leve prevê a implantação de dois estacionamentos inteligentes, conectado com os demais serviços oferecidos, pontuado como espaços que possuem "[...] tecnologias inovadoras para controle, gestão e pagamento" (PORTOLEVE, [2016?]). 
Dentro desta perspectiva encaixa-se o equilíbrio e a busca pela sustentabilidade em termos de mobilidade urbana pregadas por Okuda et al. (2012), no qual, um sistema de mobilidade urbana deve oferecer a integração dos modais de transporte existentes. Analisando no que contém o Porto Digital, é esta a perspectiva que busca ser atendida por este eixo do programa ITgreen.

Em sentido complementar, o projeto conta com o Centro de Estudos sobre Tecnologias Aplicadas à Mobilidade Urbana que atua difundindo as ações do programa (PORTO DIGITAL, [2016?]; PORTOLEVE, [2016?]). Há também proposta de debate e consolidação na temática aderente ao escopo do programa (PORTOLEVE, [2016?]).

Giffinger e Gudrun (2010) advogam que um das linhas em que segue a mobilidade inteligente busca a mobilidade, a inovação e a segurança dentre os sistemas de transporte. Em termos do eixo mobilidade urbana, ressalta-se que os três aspectos são atendidos.

\section{CONSIDERAC̣ÕES FINAIS}

Respondendo ao objetivo geral traçado para este estudo que, consistiu em explanar as ações de mobilidade urbana no programa ITgreen no Porto Digital sob a perspectiva das Smart Cities, buscou-se dentre este eixo do programa ITgreen ações que vão ao encontro do que prega o conceito e pilares das cidades inteligentes, bem como, referencial teórico que desse suporte a esta leitura.

Dentro desta perspectiva, destaca-se a percepção de que o parque tecnológico Porto Digital faz o uso inteligente das TICs, no sentido de melhoria da qualidade de vida, tanto aos cidadãos que residem na zona urbana e destinada à moradia, junto ao perímetro urbano em que ele está instalado, quanto as pessoas que circulam pelo bairro por motivo de trabalho ou, de visitação.

As práticas pelo Porto Digital implantadas por meio do programa ITgreen, seja dando destino correto ou preocupação ambiental aos resíduos tecnológicos, inclusão social por meio da acessibilidade digital ou da formação de jovens, bem como, melhoria da qualidade de vida ou de grau de especialização, conforme busca esse eixo do programa, e por fim, mas não menos importante, as soluções de mobilidade urbana, podem ser consideradas inovadoras, considerando a perceptiva social que abarcam. Para Anthony (2012) quando não permeia-se apenas o desenvolvimento financeiro, mas, envolve-se de alguma forma o social e o educacional promovem-se mecanismos para que se crie organismos autossustentáveis. 
A mobilidade urbana encaixe na perspectiva abordada por Okuda et al. (2012) e Giffinger e Gudrun (2010), quando a solução encontrada foi além do uso de TICs para um sistema de serviço dentre os modais de transporte existentes. Tais soluções abarcaram o quesito segurança, sustentabilidade e inovação, considerando os serviços comumente existentes e, as melhorias que, aparentemente, o sistema utilizado na extensão do Porto Digital oferece.

A análise fornece evidencias que as ações praticadas pelo Porto Digital e pelo programa ITgreen visam tornar o perímetro urbano em que está instalado o Porto Digital em um local/espaço/bairro inteligente. Há evidencias suficientes de que o Porto Digital preocupa-se com a melhoria da qualidade de vida de quem utiliza aquele espaço como de convivência. De acordo com isso, busca se reinventar dando forma a ideias que de alguma forma traga benefício aos cidadãos. Este estudo de caso abordou especificamente o eixo mobilidade urbana, entretanto, o Porto Digital promove outras ações que incluem inclusão social, a especialização, gestão de resíduos e, como abordado, um sistema eficiente, seguro e sustentável dos modais de transporte, conforme encontramse fundados os eixos do ITgreen.

Se o design thinking busca fugir do óbvio, focar na solução dos problemas e aumentar as possibilidades, há demonstrações dentre a atuação do Porto Digital que tais perspectivas foram e continuam sendo supridas. Da mesma forma ocorrendo com os conceitos que tangenciam as cidades inteligentes, as quais, de acordo com Al-Nasrawi, Adams e El-Zaart (2015) usam as TICs para melhorias quanto a qualidade de vida, sustentabilidade e serviços urbanos eficientes. Estas são as buscas do programa ITgreen. 


\section{REFERÊNCIAS}

AL-NASRAWI, Sukaina; ADAMS, Carl; EL-ZAART, Ali. A conceptual multidimensional model for assessing smart sustainable cities. JISTEM: Journal of Information Systems and Technology Management, São Paulo, v. 12, n. 3, p. 541-558, Sept./Dec. 2015.

AMORIM, Américo Nobre Gonçalves Ferreira; AMORIM, Tânia Nobre Gonçalves Ferreira. Iniciativas de desenvolvimento local na criação de ecossistemas inovadores em TI: o caso do Porto Digital. In: SIMPÓSIO DE EXCELÊNCIA EM GESTÃO E TECNOLOGIA DE RESENDE, 2004, Rio de Janeiro, RJ. Anais [...]. Rio de Janeiro: AEDB, 2004. v. 1, p. 1-13.

ANTHONY, Scott D. The little black book of innovation: how it works, how to do it. Boston: Harvard Business Review Press, 2012.

ANTTIROIKO, Ari-Veikko; VALKAMA, Pekka; BAILEY, Stephen J. Smart cities in the new service economy: building platforms for smart services. AI \& Society, London, v. 29, n. 3, p. 323-334, 2014.

BABBIE, Earl. The basics of social research. 6. ed. Wadsworth: Cengage Learning, 2014.

BROWN, Tim. Design thinking: uma metodologia poderosa para decretar o fim das velhas ideias. Rio de Janeiro: Elsiever, 2010.

CARAGLIU, Andrea; DEL BO, Chiara; NIJKAMP, Peter. Smart cities in Europe. Journal of Urban Technology, Abingdon, v. 18, n. 2, p. 65-82, 2011.

COSTA, Carla Silva da. Recife: The playable city: como inovação, tecnologia, criatividade e conexões podem transformar e fortalecer uma cidade. In: SEMINÁRIO NACIONAL DE PARQUES TECNOLÓGICOS E INCUBADORAS DE EMPRESAS, 24.; WORKSHOP ANPROTEC, 22., 2014, Belém. Anais [...]. Brasília: Anprotec, 2014. p. 1-17.

CRESWELL, John W. Projeto de pesquisa: métodos qualitativo, quantitativo e misto. Porto Alegre: Artmed, 2010.

GIFFINGER, Rudolph; GUDRUN, Haindlmaier. Smarter cities ranking: an effective instrument for the positioning of cities? ACE: Architecture, City and Environment, Barcelona, v. 4, n. 12, p. 7-25, feb. 2010.

ITGREEN. Mobilidade urbana. Recife, [2016?]. Disponível em: http://www. itgreen.org.br/?page_id=4. Acesso em: 16 maio 2016.

LOCKWOOD, Thomas. Design thinking: integrating innovation, customer experience, and brand value. 3rd. ed. New York: Allworth Press, 2009.

NAM, Taewoo; PARDO, Theresa A. Conceptualizing smart city with dimensions of technology, people and institutions. In: ANNUAL INTERNATIONAL CONFERENCE ON DIGITAL GOVERNMENT RESEARCH, 12., 2011, College Park. Proceedings [...]. Los Angeles: DGO, 2011. P. 282-291. 
NEIROTTI, Paolo; DE MARCO, Alberto; CAGLIANO, Anna Corinna; MANGANO, Giulio; SCORRANO, Francesco. Current trends in smart city initiatives: some stylised facts. Cities, Amsterdam, v. 38, p. 25-36, 2014.

OKUDA, Tatsuo; HIRASAWA, Shigeki; MATSUKUMA, Nobuhiko; FUKUMOTO, Takashi; SHIMURA, Akitoshi. Smart mobility for smart cities. Hitachi Review, Tokyo, v. 61, n. 3. p. 141-146, 2012.

PORTO DIGITAL. Porto Digital: parque tecnológico. Recife, [2016?]. Disponível em: http://www.portodigital.org/. Acesso em: 18 maio 2016.

PORTO DIGITAL. Porto Digital: parque tecnológico. Disponível em https:// www.portodigital.org/diferenciais/programas-e-servicos?nv1=diferenciais\&nv 2=programas-e-servicos acesso em: Março 2015

PORTOLEVE. PortoLeve. Recife, [2016?]. Disponível em: http://www. portoleve.org/. Acesso em: 20 maio 2016.

SHAMIYEH, Michael (ed.). Driving desired futures: turning design thinking into real innovation. Basel: Birkhäuser, 2014.

UCLG - UNITED CITIES AND LOCAL GOVERNMENTS. The Committee of Digital and Knowledge冈based Cities. Smart cities study: international study on the situation of ICT, innovation and knowledge in cities. Bilbao: UCLG, 2012.

UNITED NATIONS. Department of Economic and Social Affairs. World urbanization prospects: the 2014 revision: highlights. New York: United Nations, 2014. Disponível em: https://population.un.org/wup/Publications/ Files/WUP2014-Highlights.pdf. Acesso em: 9 jun. 2016.

VIANNA, Maurício; VIANNA, Ysmar; ADLER, Isabel K.; LUCENA, Brenda; RUSSO, Beatriz. Design thinking: inovação em negócios. Rio de Janeiro: MJV Press, 2012.

GIFFINGER, Rudolph; FERTNER, Christian; KRAMAR, Hans; KALASEK, Robert; PICHLER-MILANOVIĆ, Nataša; MEIJERS, Evert. Smart cities: ranking of European medium-sized cities. Vienna: Vienna University of Technology, 2007.

WEISS, Marcos César; BERNARDES, Roberto Carlos; CONSONI, Flavia Luciane. Cidades inteligentes como nova prática para o gerenciamento dos serviços e infraestruturas urbanos: a experiência da cidade de Porto Alegre. urbe: Revista Brasileira de Gestão Urbana, Curitiba, v. 7, n. 3, p. 310-324, 2015.

YIN, Robert K. Estudo de caso: planejamento e métodos. Porto Alegre: Bookman, 2010. 